\title{
Estoque de Matéria Orgânica na Superfície do Solo em Fragmentos Florestais de Mata Atlântica na APA de Petrópolis-RJ
}

\author{
Bruno do César Santos, Luana de Almeida Rangel, Evaristo de Castro Junior \\ Núcleo de Ecologia do Solo Aplicada à Geografia, Departamento de Geografia, \\ Universidade Federal do Rio de Janeiro - UFRJ
}

\section{RESUMO}

Objetivando a compreensão do estado de conservação funcional de fragmentos florestais da Mata Atlântica, na APA de Petrópolis-RJ, foram escolhidos quatro fragmentos e coletadas 13 amostras em um transecto de $130 \mathrm{~m}$ em dois verões, em 2008 e 2009. Para a camada holorgânica, foi utilizado um quadrado de $25 \times 25 \mathrm{~cm}^{2}$. O material orgânico foi separado com base em estágios de decomposição. Para a camada hemiorgânica, foram coletadas, em cada ponto, duas amostras de solo (horizonte Ai 0-3 cm e horizonte A1 3-15 cm). Posteriormente, alíquotas dos agregados do solo $>2 \mathrm{~mm}$, da TFSA, do material fino bruto (MFB) e dos agregados da camada holorgânica foram queimadas na mufla a $350^{\circ} \mathrm{C}$. Verificou-se que houve variação no estoque de Matéria Orgânica (MO) do MFB nos quatro fragmentos. Já a quantidade de MO dos agregados $>2 \mathrm{~mm}$ do $\mathrm{H}$ Ai foi inferior à do $\mathrm{H} \mathrm{A} 1$ nos dois verões.

Palavras-chave: Floresta secundária, serapilheira, decomposição.

\section{The Stock of Soil Organic Matter in Forest Fragments of the Atlantic Forest in APA Petrópolis-RJ}

\begin{abstract}
In order to understand the functional state of conservation of forest fragments of the Atlantic Forest in APA Petrópolis, State of Rio de Janeiro, four fragments were selected and 13 samples collected in a transect of $130 \mathrm{~m}$ in two consecutive summers: 2008 and 2009. A square area of $25 \times 25 \mathrm{~cm}^{2}$ was used for the holorganic layer. The organic material was separated based on stages of decomposition. For the hemiorganic layer, two samples of soil were collected at each point (Ai horizon $0-3 \mathrm{~cm}$ and $3-15 \mathrm{~cm}$ horizon A1). Subsequently, aliquots of soil aggregates $>2 \mathrm{~mm}$ of air dried soil, the fine material (MFB) and the aggregate of holorganic layer were burned in oven at $350{ }^{\circ} \mathrm{C}$. Variations in the concentration of organic matter (SOM) in MFB were observed in 4 fragments. The amount of MO aggregates $>2 \mathrm{~mm}$ HAi was lower than HA in both summers.
\end{abstract}

Keywords: Atlantic Forest, Forest Fragmentation, organic matter. 


\section{INTRODUÇÃO}

No bioma da Mata Atlântica, fragmentado e com alta diversidade de ecossistemas, a preservação do status da diversidade biológica depende da recuperação, da conservação e do uso sustentável do mosaico de fragmentos. A partir da análise do atual estado de conservação da Mata Atlântica, considerase este um dos ecossistemas mais ameaçados e devastados do planeta, mesmo representando um dos 25 hotspots de biodiversidade reconhecidos no mundo pela União Mundial para a Conservação da Natureza (Câmara, 2005).

Em ecossistemas florestais tropicais, a heterogeneidade vegetal, os tipos de solos e as características microclimáticas são diversos. É aceito que em florestas tropicais úmidas a velocidade de decomposição da matéria orgânica de superfície é rápida; contudo, o processo de fragmentação produz diferentes matrizes que comprometem o estado funcional de sistemas florestais (Kiehl, 1979).

A fragmentação florestal pode ser originada tanto pelo desmatamento, em que formações florestais estão circundadas por diferentes tipos de habitats não florestados (Castro Junior, 2002), quanto pode ocorrer quando um ecossistema é subdividido pela ação do homem ou por perturbações naturais, resultando em uma paisagem na qual permanecem alguns fragmentos da cobertura vegetal original, inseridos em uma matriz diferente (Kindel, 2001).

Os fragmentos que hoje existem estão, em sua maioria, inseridos em matrizes de diferentes tipos, os quais se configuram muitas vezes como barreiras para a dispersão de espécies, pois o tempo de isolamento, determinado pela distância entre fragmentos vizinhos, as características do ambiente entre os fragmentos e o grau de conectividade são determinantes na resposta da biota à fragmentação (Kindel, 2001).

Para avaliar a diversidade no nível de ecossistemas, é possível utilizar Indicadores Funcionais Globais, os quais são variáveis que sintetizam o funcionamento do ecossistema, notadamente definidos pelos dois maiores processos: produtividade e decomposição (Garay \& Silva, 1995).

Quanto maior o estoque de matéria orgânica do solo, mais lento é o processo de decomposição e maior é o aporte de material que chega ao solo. Logo, o estoque de matéria orgânica de superfície do solo é considerado como um indicador funcional da decomposição em sistemas florestais.

Em sistemas florestais, o estoque de matéria orgânica de superfície, denominado forma de húmus, tem uma relação com o padrão do estado de evolução funcional do subsistema de decomposição (Haag, 1985). Assim, interações entre os processos bióticos (vegetação, fauna edáfica e organismos saprófagos - fungos e bactérias) e abióticos (clima, relevo, topografia, solo e umidade, entre outros) controlam variações observadas nas formas de húmus. Perturbações funcionais na dinâmica de matéria orgânica nesses sistemas florestais, por exemplo, em decorrência de diferentes tipos de manejo, podem ser evidenciadas por variações de padrões de evolução sucessional do estoque (Primavesi, 1987).

Nos sistemas de florestas tropicais, a ciclagem de nutrientes ocorre principalmente nas camadas que compõem a serapilheira na interface com o solo. Didham (1998) afirma que a decomposição da serapilheira gera um ciclo de nutrientes vital para a produtividade das florestas. Logo, o funcionamento do subsistema de decomposição é o maior responsável pela manutenção do ecossistema (Poggiani et al., 1987).

Com isso, o topo do solo possui grande papel no processo de ciclagem de nutrientes em sistemas florestais tropicais, pois é nele que se encontram os maiores valores de carbono $(\mathrm{C})$ e nitrogênio $(\mathrm{N})$ (Kindel \& Garay, 2002), assim como boa parte da vida biológica responsável pela decomposição da matéria orgânica (Silva, 1998).

Como no Brasil ainda são escassos trabalhos relacionados ao tema proposto, é necessário aprofundar estudos para entender melhor os processos de decomposição de um sistema de floresta ombrófila, comparando os estoques de matéria orgânica em diferentes fragmentos florestais circundados por matrizes distintas. Assim, as variações da acumulação da matéria orgânica de superfície e a sua decomposição, em síntese, podem ser um indicador de perturbações na ciclagem de nutrientes nos sistemas ecológicos.

Com isso, o estoque de matéria orgânica do material fino dos agregados da camada holorgânica, 
da terra fina seca ao ar (TFSA) e dos agregados do horizonte hemiorgânico (A e Ai) é interpretado como um indicador da dinâmica biológica do processo físico-químico da decomposição, que permite analisar melhor a dinâmica da ciclagem de nutrientes nestes ambientes (Didham, 1998).

Partindo da hipótese de que diferentes matrizes podem influenciar negativamente $\mathrm{o}$ processo de decomposição de um fragmento florestal e, consequentemente, no estoque de matéria orgânica em um determinado fragmento, buscou-se, por meio do presente trabalho, entender os diferentes processos de decomposição em fragmentos florestais na APA-Petrópolis, no Estado do Rio de Janeiro.

Para isso, elaborou-se uma análise comparativa dos estoques de matéria orgânica do material em diferentes compartimentos da camada superficial do solo/serapilheira, em quatro fragmentos florestais de floresta ombrófila montana, submetidos a histórias singulares de manejo, localizados em diferentes matrizes geográficas inseridas na APA de PetrópolisRJ, em contextos rural, urbano, silvestre e rururbano.

Portanto, tem-se como objetivo, a partir da realização de comparações interfragmentos e intrafragmentos do estoque de matéria orgânica (MO) de superfície do solo, e comparando os verões de 2008 e de 2009, entender melhor o processo de decomposição e de interferência sobre o estado sucessional de fragmentos florestais, influenciado por diferentes usos.

\section{MATERIAL E MÉTODOS}

A pesquisa se desenvolveu em fragmentos florestais remanescentes da Mata Atlântica, localizados na APA-Petrópolis, município de Petrópolis, Estado do Rio de Janeiro. A APAPetrópolis representa um mosaico de Unidades de Conservação do Corredor Central Fluminense do Bioma Mata Atlântica (Sistema Nacional de Unidades de Conservação) e tem um papel importante em garantir o fluxo gênico, pois encerra uma gama variada de tipos de fragmentos florestais entre a Reserva Biológica - Rebio - Tinguá e o Parnaso (Parque Nacional da Serra dos Órgãos) (Figura 1).

A APA-Petrópolis está inserida no Bioma Mata Atlântica, tendo como domínio a Floresta Ombrófila Densa (IBGE, 1992). Em cada local, dependendo dos solos existentes, do potencial hídrico médio do solo e dos mesoclimas circundantes, poderão ser observadas algumas formações vegetacionais características. Como, por exemplo, florestas densas, que apresentam diversidade de espécies botânicas e da fauna em sua expressão original, com estágios variados da regeneração natural; comunidade rupestre, que possui expressões vegetacionais típicas dos afloramentos rochosos, onde inexistem solos propriamente ditos ou restam apenas finas camadas de turfa sobre a pedra; campos de altitude, que estão intimamente ligados aos litossolos altitudinais, e matas nebulares ou nuvígenas, que são matas de

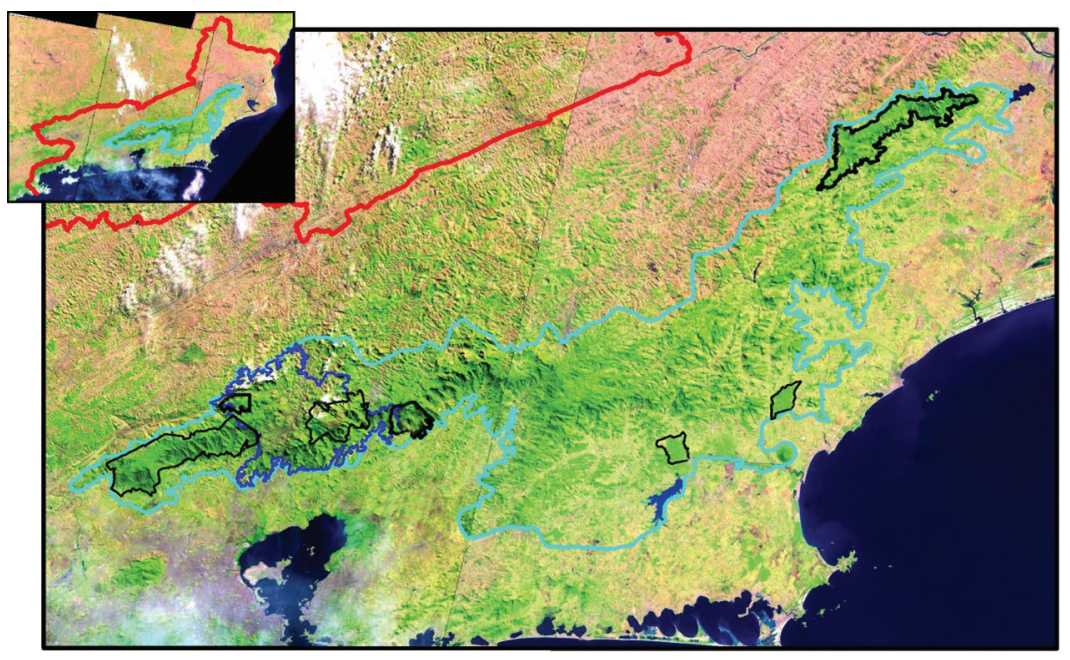

Figura1. Localização da APA-Petrópolis.

Figure 1. Localization of APA-Petrópolis. 
aspecto xerofítico e esclerófilo, típicas das beiradas dos mais altos morros da serra.

A porção sul da APA-Petrópolis corresponde às escarpas de falha da Serra do Mar, situada na faixa de dobramento remobilizado formado pelas escarpas e reversos desta Serra. Na parte sul da APA-Petrópolis, encontram-se escarpas de falha da Serra do Mar. Toda essa área drena para a Baía de Guanabara e grande parte será incorporada ao Parnaso.

Do ponto de vista da estrutura geológica, a região é complexa, com extensos falhamentos escalonados, já amplamente dissecados pelos processos erosivos, formando os contrafortes da Serra do Mar. O conjunto rochoso é constituído de bloco de falha com dominância do granito gnaisse. As rochas gnáissicas ocorrem associadas a pequenos maciços de granitos compostos, assim como migmatitos. Apresenta declividades expressivas e variação de altitude que vai da cota de 100 até 1919 m (Pico da Maria Comprida).

Quanto aos solos, há predominância de Latossolos (Amarelo, Vermelho e Vermelho Amarelo, além de diversas combinações entre eles), além de Neossolos Litólicos e Cambissolos nas áreas de maior altitude, e Organossolos e Gleissolos nas regiões mais baixas, sobretudo em fundos de vale, na beira de rios. (Embrapa, 1990) Os Latossolos são solos bastante lixiviados, com predominância de argilominerais 1:1 e óxidos de ferro e alumínio, que tornam estes solos de reduzida fertilidade. Apresentam perfis profundos, pouco diferenciados, sendo pouco nítido o contraste entre seus horizontes. A percentagem de matéria orgânica destes solos é maior em superfície e se reduz conforme a profundidade (Embrapa, 2006)

Os solos hidromórficos são mais frequentes na parte sul, nas porções mais baixas da Unidade de Conservação - UC, e na porção central, onde o relevo mais suave possibilita um maior acúmulo de água nos solos.

O fragmento I (Fazenda Itaipava - FI) (Figura 2) está localizado numa área dominada por floresta secundária densa e apresenta forte domínio de gramíneas e espécies arbustivas. Boa parte do fragmento esta sendo loteada, com a construção de

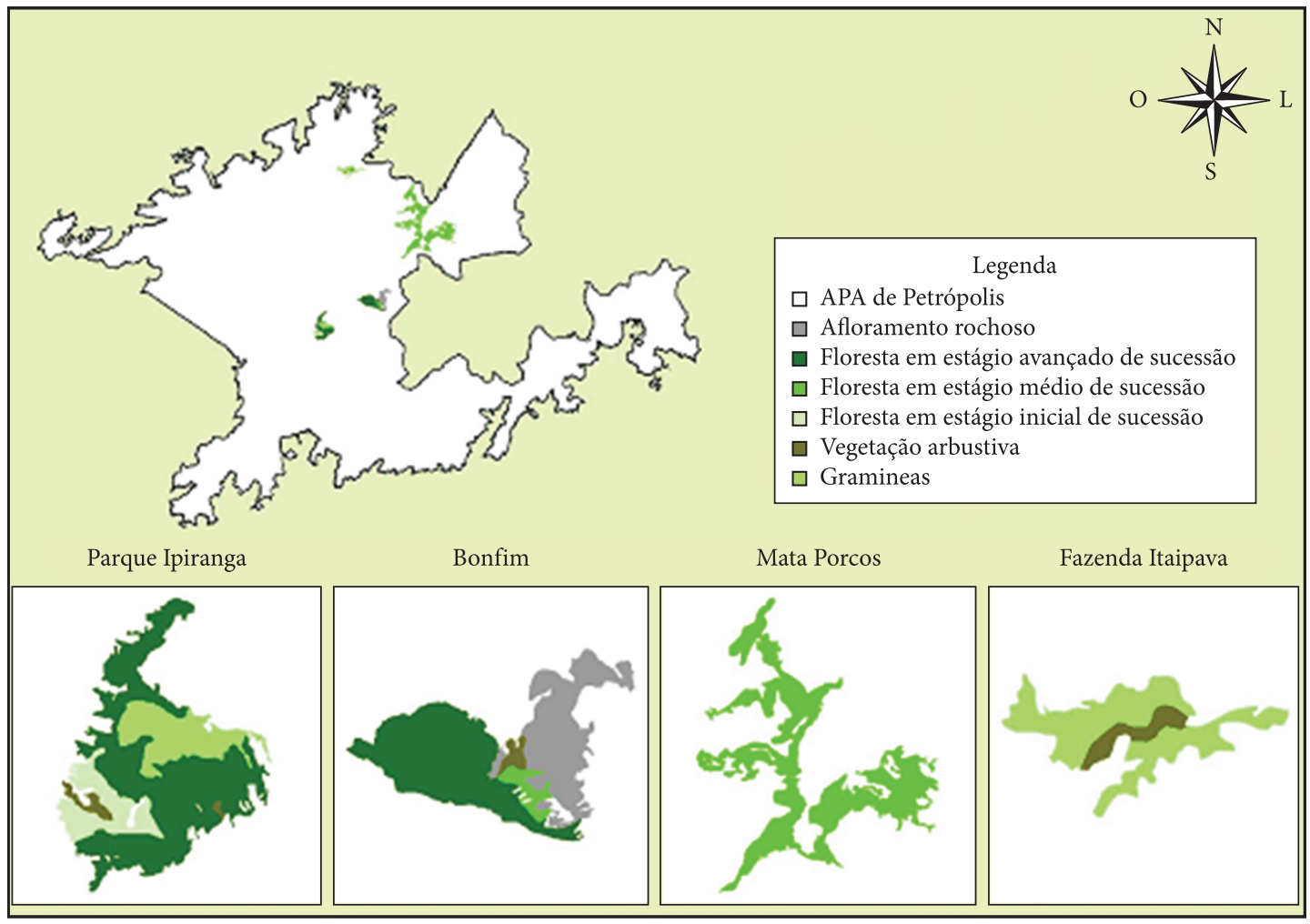

Figura 2. Localização dos fragmentos na APA- Petrópolis.

Figure 2. Localization of fragments in APA-Petrópolis. 
condomínios e casas. É possível dizer que o fragmento esta inserido em uma matriz que se assemelha a uma área rururbana (uma fisionomia rural, porém sem o uso econômico de atividades agrícolas).

No fragmento II (Parque Ipiranga - PI) (Figura 2), trata-se de um Parque Municipal de proteção permanente (SNUC Lei 9.985), parque que é utilizado para trilhas turísticas, também existindo ocupações irregulares e tendo como vegetação dominante deste fragmento florestas em estágio sucessional avançado, estágio inicial de sucessão, gramíneas e vegetação arbustiva. Localizado no centro da Cidade de Petrópolis, sua matriz é urbana.

O fragmento III (Bonfim - BO) (Figura 2) está localizado numa área periférica de Petrópolis; a vegetação dominante é composta por florestas em estágio avançado e médio de sucessão, assim como por vegetação arbustiva. No entanto, este fragmento apresenta um controle litológico na sua porção nordeste, com embasamento gnáissico aflorante. Grande parte do fragmento tem sua matriz circundante por floresta natural, porém a parte oeste do fragmento tem ação antrópica, sendo a matriz circundante rural.

O fragmento IV (Condomínio Mata Porcos MP) (Figura 2) tem como vegetação dominante uma floresta em estágio médio de sucessão, dentre os outros fragmentos. Este fragmento apresenta uma forma mais alongada, caracterizando menor distância entre as bordas. Este fragmento é circundado por áreas rurais de produção familiar e áreas de urbanização de baixa densidade; portanto, pode-se considerá-lo como tendo uma matriz circundante rururbana.

Em cada fragmento, foi estabelecido um transecto de $130 \mathrm{~m}$ de comprimento a partir de $20 \mathrm{~m}$ da borda de trilhas no interior do fragmento (para evitar o efeito de borda). Os transectos foram estabelecidos em encosta retilínea. A cada $10 \mathrm{~m}$, ao longo dos transectos, foi estabelecido um ponto amostral, no qual foi coletada uma amostra aleatória, totalizando 13 amostras por transecto.

Para o estudo da camada Holorgânica, foi coletada uma amostra em cada ponto, em um quadrado de $25 \times 25 \mathrm{~cm}^{2}$. O material orgânico de superfície foi separado em laboratório, com base em seus estágios de decomposição, em camadas sucessionais no processo de decomposição; também foi separado o material fino bruto - MFB (obtido a partir da peneiragem com malha de $2 \mathrm{~mm}$ ) e os agregados $>2 \mathrm{~mm}$.

Já em relação à camada superficial do solo composto por material mineral e matéria orgânica humificada (Horizonte Hemiorgânico), foram coletadas, em cada ponto, duas amostras deformadas de solo (horizonte Ai, 0-3 cm e horizonte A, 3-15 cm). As amostras do horizonte Ai foram coletadas também com o auxílio de um quadrado de $25 \times 25 \mathrm{~cm}^{2}$; já as amostras do horizonte A foram coletadas com um cilindro de $13 \mathrm{~cm}$ de altura e $10 \mathrm{~cm}$ de diâmetro. As amostras foram submetidas a análises físicas, pelas quais foi calculada a percentagem de agregados $>2 \mathrm{~mm}$ pelo método por via seca (Embrapa, 1997) e separada uma parte de TFSA (terra fina seca ao ar) do Ai e do A1, para análise da matéria orgânica.

Posteriormente, alíquotas dos agregados do solo $>2 \mathrm{~mm}$ do Horizonte Ai e A, da TFSA, do MFB e dos agregados da camada holorgânica foram queimados na mufla por 16 horas a $350{ }^{\circ} \mathrm{C}$ (Embrapa, 1997); a partir desse procedimento, foi encontrado o valor total do estoque de matéria orgânica do material fino e dos agregados. Para isso, foram pesados $10 \mathrm{~g} \mathrm{em}$ cada alíquota; depois de retirados da mufla, foram pesados novamente e feita a diferença segundo a fórmula: P1 - P2 = PMO, em que P1 é o Peso Conhecido (10 g), P2 é o peso depois da queima e PMO é o peso que foi queimado, no caso, de matéria orgânica.

$\mathrm{Na}$ análise dos dados, foram calculadas a média e o desvio padrão da média como expressão sintética dos resultados. Para as comparações entre os mesmos fragmentos nos dois verões, foi empregado o teste de U de Mann-Whitney; para as comparações entre os quatro fragmentos, são empregados os testes de Kruskal-Wallis. Ambos os testes estatísticos constituem-se em testes não paramétricos, por comparação das medianas (Triola, 2008).

\section{RESULTADOS E DISCUSSÃO}

A quantidade de matéria orgânica no material fino bruto (MFB) e a relação $\mathrm{C} / \mathrm{N}$ do horizonte $\mathrm{A}$ são a base para a diferenciação das velocidades de decomposição da matéria orgânica de superfície 
(Kindel \& Garay, 2002). Swift et al. (1979) relatam que, em uma região tropical e intertropical, a temperatura desempenha um papel de fundamental importância na dinâmica da decomposição da matéria orgânica de solos, quando a umidade não é fator limitante.

$\mathrm{Na}$ amostragem de 2008, o fragmento I possui maior quantidade de matéria orgânica tanto nos agregados dos horizontes A e Ai quanto na TFSA do $\mathrm{Ai}$, em relação aos outros fragmentos. Destacase que o fragmento I é significativamente igual ao fragmento IV na quantidade de MO encontrada nos agregados do horizonte A. O fragmento III também apresenta elevado estoque de matéria orgânica nos agregados da serapilheira (camada holorgânica), apesar de não possuir diferença significativa segundo o teste estatístico (Tabela 1).

Já o fragmento II difere significativamente na quantidade de matéria orgânica no $\mathrm{MFB}$ da camada holorgânica dos demais fragmentos. Desta maneira, é possível que o fragmento II tenha uma taxa de decomposição mais lenta em relação aos outros, devido ao grande acúmulo de material orgânico na camada holorgânica. Isso pode estar ocorrendo devido a algum estresse (implementação de infraestrutura urbana, como casas e construções) pelo qual o fragmento possa estar passando devido à influência da matriz circundante (urbana). Tal quantidade de MO seguiu uma tendência do fragmento mais perturbado para o menos degradado - FII $>$ FIII $>$ F1 $>$ FIV.
Problemas no processo de ciclagem de nutrientes, como a deficiência da troca de cátions, por exemplo, podem reter a matéria orgânica na serapilheira e diminuir a quantidade de C para o solo (Franzluebbers, 2002).

Já o fragmento III mostra uma velocidade de decomposição acelerada, pois apresenta baixa quantidade de $\mathrm{MO}$ nos agregados do $\mathrm{A}$ e do Ai. Essas características podem estar indicando um bom estado de conservação deste sistema frente à fragmentação, o que permite melhor dinâmica de raízes e captação de água.

A grande concentração de agregados de frações grandes presentes no topo dos solos de florestas tropicais, identificada por (Kindel \& Garay, 2002), mostra a importância do desenvolvimento de estudos que abordem essa característica peculiar dos solos desses ambientes..

Não houve diferença significativa na quantidade de MO dos agregados de serapilheira entre os fragmentos no ano de 2008.

Para a coleta no verão de 2009, não houve diferença significativa no estoque de matéria orgânica no MFB da camada holorgânica entre os fragmentos. O fragmento II apresentou o menor valor, diferentemente de 2008 (Tabela 2).

O fragmento III apresentou diferença significativa em relação aos outros fragmentos, com baixo estoque de MO nos agregados da serapilheira, diferentemente do ano anterior de 2008.

Tabela 1. Comparação do estoque de matéria orgânica nos diferentes compartimentos dos fragmentos no verão de 2008. Os números correspondem à mediana. Letras diferentes na mesma linha demonstram diferença significativa pelo teste de Kruskal-Wallis. Quando não apresenta letra, indica que não houve diferença significativa.

Table 1. Comparison of the stock of organic matter in the soil between fragments in the summer of 2008. Numbers correspond to mean \pm standard deviation. Different letters in the same row show significant differences by KruskalWallis. When we do not presents letter means no significant difference.

\begin{tabular}{|c|c|c|c|c|}
\hline \multicolumn{5}{|c|}{ Coleta 2008} \\
\hline \multirow{2}{*}{ Matéria orgânica em t.ha ${ }^{-1}$} & Fragmento I & Fragmento II & Fragmento III & Fragmento IV \\
\hline & $(n=13)$ & $(n=13)$ & $(n=13)$ & $(n=13)$ \\
\hline Material fino & $1,89^{\mathrm{B}}$ & $3,40^{\mathrm{A}}$ & $2,67^{\mathrm{B}}$ & $1,64^{\mathrm{B}}$ \\
\hline Agregados serapilheira & 0,38 & 0,42 & 1,07 & 0,22 \\
\hline Agregados $>2 \mathrm{~mm}(\mathrm{Ai})$ & 3,26 & 3,09 & 2,54 & 2,59 \\
\hline TFSA (Ai) & 8,37 & 7,15 & 8,17 & 6,59 \\
\hline Agregados $>2 \mathrm{~mm}(\mathrm{~A} 1)$ & $4,92^{\mathrm{B}}$ & $3,96^{\mathrm{AB}}$ & $3,01^{\mathrm{A}}$ & $4,38^{\mathrm{B}}$ \\
\hline TFSA (A1) & 4,85 & 4,35 & 6,07 & 4,53 \\
\hline
\end{tabular}


Já em relação aos agregados do Horizonte Ai, observa-se que o estoque de MO no fragmento I difere dos fragmentos II e III; porém, apresenta semelhança com o fragmento IV. Já o estoque de MO para os agregados do A foi menor para o fragmento III e os outros fragmentos foram semelhantes estatisticamente.

O estoque de matéria orgânica no MFB, de TFSA (Ai) e TFSA (A), não apresentou diferença estatística significativa para o verão de 2009.

Verifica-se que, em 2008, os valores de matéria orgânica de solo na TFSA foram maiores no horizonte Ai em comparação com o horizonte A; esse fato ocorre porque há uma maior entrada de MO em decomposição vinda do material fino, como dito anteriormente. Esse processo ocorre porque o horizonte $\mathrm{Ai}$ (interface) retém a maior disponibilidade de nutrientes e sustenta as mais altas taxas de fertilidade; também, porque o horizonte A é mais profundo, onde ocorre mineralização de alguns materiais, havendo maior quantidade de argila vinda de horizontes acima, em razão do processo de translocação.

Ao comparar o estoque de MO no verão de 2008 e no verão de 2009 (Tabela 3) no fragmento I, observou-se que nos Agregados $>2 \mathrm{~mm}$ (A) ocorreu uma variação de um ano para o outro, demonstrando que o estoque está diminuindo.

Tabela 2. Comparação do estoque de matéria orgânica do solo, entre os fragmentos no verão de 2009. Os números correspondem à mediana. Letras diferentes na mesma linha demonstram diferença significativa pelo teste de Kruskal-Wallis. Quando não apresenta letra, indica que não houve diferença significativa.

Table 2. Comparison of the stock of organic matter in the soil between fragments in the summer of 2009. Numbers correspond to median. Different letters in the same row show significant differences by Kruskal-Wallis. When we do not presents letter means no significant difference.

\begin{tabular}{|c|c|c|c|c|}
\hline \multicolumn{5}{|c|}{ Coleta 2009} \\
\hline \multirow{2}{*}{ Matéria orgânica em t.ha ${ }^{-1}$} & Fragmento I & Fragmento II & Fragmento III & Fragmento IV \\
\hline & $(n=13)$ & $(n=13)$ & $(n=13)$ & $(n=13)$ \\
\hline Material fino & 1,83 & 1,06 & 1,69 & 1,21 \\
\hline Agregados serapilheira & $0,48^{\mathrm{A}}$ & $0,14^{\mathrm{A}}$ & $0,05^{\mathrm{B}}$ & $0,11^{\mathrm{A}}$ \\
\hline Agregados $>2 \mathrm{~mm}(\mathrm{Ai})$ & $3,64^{\mathrm{A}}$ & $2,19^{\mathrm{B}}$ & $2,44^{\mathrm{B}}$ & $2,73^{\mathrm{AB}}$ \\
\hline TFSA (Ai) & 7,36 & 10,03 & 8,88 & 7,46 \\
\hline Agregados $>2 \mathrm{~mm}(\mathrm{~A})$ & $5,18^{\mathrm{A}}$ & $4,24^{\mathrm{A}}$ & $3,59^{\mathrm{B}}$ & $4,26^{\mathrm{A}}$ \\
\hline TFSA (A) & 5,49 & 5,57 & 5,49 & 5,57 \\
\hline
\end{tabular}

Tabela 3. Comparação do estoque de matéria orgânica do solo, entre os dois períodos de coleta. Valores correspondem à mediana. Quando existe diferença significativa pelo teste U de Mann-Whitney entre os dois períodos de coleta dentro de um mesmo fragmento, o teste aparece na coluna referente ao ano de 2008. Quando não apresenta letra, indica que não houve diferença significativa.

Table 3. Comparison of the stock of organic matter in the soil between summer of 2008 and summer of 2009. Numbers correspond to median. When there is significant difference test by Mann-Whitney U between the two collection periods within the same fragment, the test appears in the column for the year 2008. When we do not presents letter means no significant difference.

\begin{tabular}{|c|c|c|c|c|c|c|c|c|}
\hline \multicolumn{9}{|c|}{ Coleta 2008 e 2009} \\
\hline \multirow{2}{*}{$\begin{array}{c}\text { Matéria orgânica } \\
\text { em t.ha }^{-1}\end{array}$} & \multicolumn{2}{|c|}{ Fragmento I } & \multicolumn{2}{|c|}{ Fragmento II } & \multicolumn{2}{|c|}{ Fragmento III } & \multicolumn{2}{|c|}{ Fragmento IV } \\
\hline & 2008 & 2009 & 2008 & 2009 & 2008 & 2009 & 2008 & 2009 \\
\hline Material fino & 1,83 & 1,89 & $1,06^{*}$ & 3,40 & 1,69 & 2,67 & 1,21 & 1,64 \\
\hline Agregados serapilheira & 0,48 & 0,38 & $0,14^{\star}$ & 0,42 & $0,05^{*}$ & 1,07 & 0,11 & 0,22 \\
\hline Agregados > 2 mm (Ai) & 3,64 & 3,26 & 2,19 & 3,09 & 2,44 & 2,54 & 2,73 & 2,59 \\
\hline TFSA (Ai) & 7,36 & 8,37 & 10,03 & 7,15 & 8,88 & 8,17 & 7,46 & 6,59 \\
\hline Agregados $>2 \mathrm{~mm}(\mathrm{~A})$ & $5,18^{*}$ & 4,92 & 4,24 & 3,96 & 3,59 & 3,01 & 4,26 & 4,38 \\
\hline TFSA (A) & 5,49 & 4,85 & 5,57 & 4,35 & 5,49 & 6,07 & $5,57^{*}$ & 4,53 \\
\hline
\end{tabular}


Já para o Fragmento II, ocorreu diferença significativa no MFB entre os períodos de amostragem e entre os agregados da serapilheira, demonstrando que houve variação no estoque da camada holorgânica.

No fragmento III, verificou-se diferença significativa apenas no estoque de $\mathrm{MO}$ dos agregados da serapilheira, o que pode estar acontecendo por causa da influência da atividade da microfauna do solo.

O fragmento IV apresentou diferença significativa em relação ao estoque de MO na TFSA do horizonte A, comparando os dois períodos de coleta, o que pode estar ocorrendo porque a matriz circundante (rururbana) está influenciando na dinâmica de matéria orgânica no fragmento.

Para os dois períodos de coleta, o comportamento de MO na TFSA foi inversamente proporcional ao dos agregados; tal fato ocorre porque os agregados do solo possuem grande quantidade de argila e outros materiais que provocam a cimentação dessas partículas. Foram encontrados os maiores valores de estoque de $\mathrm{MO}$ no horizonte $\mathrm{Ai}$, porque é nesse horizonte de interface que ocorre o maior aporte de matéria orgânica sendo decomposta pelo sistema, havendo maior dinâmica da fauna do solo e de microrganismos decompositores.

\section{CONCLUSÕES}

Verificou-se que o estoque de matéria orgânica do solo variou para os fragmentos nos dois períodos de coleta. O fragmento que mais apresentou variações no estoque foi o fragmento II, que possui a matriz circundante urbana. Porém, essa variação ocorreu positivamente de 2008 para 2009, pois os estoques de $\mathrm{MFB}$, agregados da serapilheira e agregados $>2 \mathrm{~mm}$ (Ai) aumentaram.

$\mathrm{O}$ fragmento I mostrou-se mais afetado pela matriz circundante, pois o estoque de $\mathrm{MO}$ nos agregados da serapilheira, agregados $>2 \mathrm{~mm}$ (Ai), agregados $>2 \mathrm{~mm}$ (A) e TFSA (A) diminui de 2008 para 2009. Logo, é possível afirmar que a matriz rururbana está influenciando negativamente o estoque de matéria orgânica do solo.

Em relação ao fragmento III, observou-se que o estoque de MO na camada holorgânica aumentou de um ano para o outro, principalmente para os agregados da serapilheira, demonstrando que a agregação no topo do solo não está sendo influenciada negativamente pelas atividades desenvolvidas no entorno desse fragmento.

Por fim, o fragmento IV apresentou diferença significativa no estoque de MO para a TFSA (A), ocorrendo diminuição do estoque de um verão para o outro.

Os fragmentos II, III e IV tiveram aumento do estoque de MO na camada holorgânica de um período de coleta para o outro.

Portanto, como a decomposição é de fundamental importância para a manutenção do ecossistema, qualquer alteração na dinâmica desse subsistema por meio do estoque de matéria orgânica do solo, como a influência das matrizes circundantes, pode acarretar consequências nocivas à preservação do meio ambiente e alterações na biodiversidade dos fragmentos florestais.

\section{AGRADECIMENTOS}

Ao Fundo de Amparo à Pesquisa do Estado do Rio de Janeiro (FAPERJ), pelo financiamento da pesquisa, e ao Núcleo de Ecologia do Solo Aplicada à Geografia - NESAG da UFRJ, pela utilização do laboratório.

\section{STATUS DE SUBMISSÃO}

Recebido: 07/07/2011

Aceito em: 08/09/2011

Resumo publicado online: 15/09/2011

Artigo completo publicado: 30/09/2011

\section{AUTOR(ES) PARA CORRESPONDÊNCIA}

\section{Luana de Almeida Rangel}

Núcleo de Ecologia do Solo Aplicada à Geografia, Departamento de Geografia, Uiversidade Federal do Rio de Janeiro - UFRJ, Rua Athos da Silveira Ramos, 274, Prédio CCMN, Bloco G, Ilha do Fundão, CEP 21941-916, Rio de Janeiro, RJ, Brasil, e-mail: luarangel24@ig.com.br 


\section{REFERÊNCIAS}

Câmara IG. Breve história da conservação da Mata Atlântica. In: Galindo-Leal C, Câmara IG, editors. Mata Atlântica: biodiversidade, ameaças e perspectivas. São Paulo: Fundação SOS Mata Atlântica; Belo Horizonte: Conservação Internacional; 2005.

Castro Junior E. Valor indicador da fauna de macroartrópodes edáficos em fragmentos primários e secundários do ecossistema de florestas de tabuleiros, ES [tese]. Rio de Janeiro: Universidade Federal do Rio de Janeiro; 2002.

Didham RK. Altered Leaf-Litter decomposition raters in tropical Forest fragments. Oecologia 1998; 116:317-406. http://dx.doi.org/10.1007/s004420050603

Embrapa. Serviço Nacional de Levantamento e Conservação do Solo - SNLCS. Manual de Métodos de análise de solos. Rio de Janeiro: EMBRAPA/SNLCS; 1997. v. 1.

Embrapa. Sistema brasileiro de classificação de solos. 2. ed. Rio de Janeiro: Embrapa Solos, 2006.

Franzluebbers AJ. Soil organic matter stratification ratio as an indicator of soil quality. Soil and Tillage Research 2002; 66(2):95-106. http://dx.doi.org/10.1016/S01671987(02)00018-1

Garay I, Silva BAO. Húmus Florestais: síntese e diagnóstico das interrelações vegetação solo. In: Esteves F, editors. Oecologia Brasilienses. Rio de Janeiro; 1995.

Haag HP. Ciclagem de nutrientes em florestas tropicais. Campinas: Fundação Cargill; 1985. 114 p.
Jordan CF. Amazon rain forest. Amsterdam 1982; 70:394-401.

Kiehl E J. Manual de Edafologia: Relações solo-planta. São Paulo: Editora Agronômica Ceres; 1979.

Kindel A. A fragmentação Real: Heterogeneidade de remanescentes florestais e valor indicador das formas de húmus [tese]. Rio de Janeiro: Universidade Federal do Rio de Janeiro; 2001.

Kindel A, Garay I. Humus forms in ecosystems of the Atlantic Forest, Brazil. Geoderma 2002; 108:101-118. http://dx.doi.org/10.1016/S0016-7061(02)00126-X

Poggiani, F.; Zamberlan, E.; Monteiro Jr. \& Gava, I. C. Quantificação de deposição de folhedo em talhões experimentais de Pinu Taeda, Eucalyptus viminalis e mimosa scabrella plantados em uma área degradada pela mineração de xisto betuminoso. IPEF, Piracicaba, v. 37, p. 21-29. 1987

Primavesi A. O manejo ecológico do solo. São Paulo: Nobel; 1987. 541 p.

Silva, RF. Roça caiçara: dinâmica de nutrientes, propriedades físicas e fauna do solo em um ciclo de cultura. Tese de Doutorado. UFRRJ-Seropédica, Rio de Janeiro. 1998.

Swift MJ, Heal OW, Anderson JM. Decomposition in terrestrial ecosystems. Berkeley: University of California; 1979.

Triola MF. Introdução à Estatística. Rio de Janeiro: LTC; 2008.

Veloso, HP., Rangel Filho, AL., LIMA, JC. Classificação da Vegetação Brasileira adaptada a um sistema universal. IBGE, 1992. 